\title{
EFECTO DE LA LEY DE OPA EN EL RETORNO DE LA ACCIÓN EN CHILE
}

\author{
Darcy Fuenzalida O'Shee*
}

Universidad Técnica Federico Santa María, Chile

Mauricio Nash Sarquis

Universidad Técnica Federico Santa María, Chile

(Recibido 4 de junio 2003, aceptado 26 de septiembre 2003)

\section{Resumen}

La evidencia internacional, principalmente estadounidense, ha encontrado retornos anormales positivos para la empresa que es objeto de una oferta pública de acciones (OPA) al momento de su anuncio. Esta Investigación, tiene como objetivo contrastar la evidencia internacional con la chilena, y analizar el efecto de la Ley de OPA en el retorno de una acción. Para ello, se aplicarán criterios de selección a la totalidad de las OPA's en Chile en el periodo 1995-2002, seleccionándose catorce empresas. Se encontró evidencia en Chile, de que al momento de anunciarse una OPA, se generan las expectativas que dan como resultado retornos anormales positivos el día del anuncio, y que los retornos son menores cuando existe Ley de OPA.

\section{Abstract}

Several studies, mainly made in The United States, have found abnormally positive returns for the company which is the target of a "Tender Offer", at the moment of the announcement. The aim of this research is to compare the international evidence with the chilean one. For this purpose, we studied fourteen important chilean public companies where "Tender Offers" have taken place, between 1995 and 2002. Also we studied the effect that a Law of Tender Offers has in the returns of a given share. Thus, we can finally conclude, that yes there is evidence in Chile, that at the moment a Tender Offer is made public, it generates expectations, producing as a result abnormal positive retuns, on the day of the announcement. These abnormal positive returns are lower in the case of public companies operating under the Law of Tender Offers.

Clasificación JEL: C40, C52, C91, C93

Palabras clave: Tender Offer (OPA), Modelos estadísticos, Periodo de Evento, Periodo de Pre-Evento, Prueba de error individual, Prueba de error acumulado

* Departamento de Industrias. Universidad Técnica Federico Santa María. Avenida España 1680, Casilla 110-V, Valparaíso, Chile. Teléfono: +(32) 654272. Correo electrónico: darcy.fuenzalida@usm.cl

Los autores desean agradecer a dos dictaminadores anónimos sus valiosas observaciones. 


\section{Introducción}

Este artículo tiene por objetivo contrastar la evidencia estadounidense, la cual sugiere la presencia de retornos anormales positivos, para las empresas que son objeto de un "tender offer" u OPA al momento de anunciarse éste, con la chilena, y por otro, lado analizar el efecto de una legislación de OPA en el retorno de la acción. Para esto, se aplicaron algunos criterios de selección a la totalidad de OPA's que han ocurrido entre los años de 1995 y el de 2002, y se construyó una muestra de catorce sociedades anónimas chilenas que han materializado una OPA. Lo anterior, con el fin de poder responder las siguientes preguntas: ¿En Chile, las empresas que son objeto de una OPA, presentan retornos anormales positivos al momento del anuncio? ¿Qué razones explicarían las diferencias en la significancia de los retornos en la acción de las empresas que son objeto de una OPA? y ¿Cuál es el efecto de la ley de OPA en el retorno de la acción?

En la sección 1, se presentará la evidencia empírica de un "takeover". En la sección 2, se explicará la Ley de OPA Chilena. En la sección 3, se proporcionará una descripción de la estimación de retornos anormales. Por otra parte, en la sección 4, se tratarán los resultados obtenidos a partir de la muestra y finalmente, en la sección 5 se presentan las conclusiones.

\section{Teoría y evidencia empírica respecto a los "takeover"}

Un "takeover" es un acuerdo o actividad de intercambio entre dos partes, accionistas controladores de una empresa objetivo y accionistas controladores de una empresa oferente, es decir, aquella que desea adquirir una firma objetivo con el fin de poseer una unidad económica distinta a la existente.

Algunos estudios, principalmente del mercado estadounidense, sobre los "takeover" (adquisición o fusión de una empresa) son: Dodd y Ruback (1977) estudiaron 172 empresas que se transaban en la bolsa de New York, cubriendo un periodo entre 1958 y 1976 . El objetivo de ese estudio era analizar la prima obtenida por las empresas "target" (objetivo) ante el anuncio de un "tender offer". Este estudio incluía operaciones exitosas y no exitosas. Empleando el modelo de mercado, los autores encontraron que los retornos anormales de las empresas objetivo que fueron adquiridas a través de ofertas exitosas, ascendía a un $21 \%$. Para las ofertas no exitosas el retorno fue de $19 \%$.

Jensen y Ruback (1983) efectuaron 13 estudios entre los años 1977 y 1983, concluyendo que los "takeover" incluidos en la muestra obtuvieron retornos anormales positivos entre un $16 \%$ y un $30 \%$. Por otra parte, Bradley, Desai y Kim (1983) encontraron un premio para las acciones de las empresas objetivo de un "tender offer" de entre un $23 \%$ y un $60 \%$ en el NYSE.

Jarrell y Poulsen (1987), estudiaron 663 casos de "tender offers" exitosas entre 1962 y 1985, concluyendo que las acciones de las empresas "target" promediaban retornos anormales positivos de $30 \%$. Esto es consistente con el estudio mencionado anteriormente, efectuado por Jensen y Ruback (1983). De igual forma, Asquith (1988) encontró un premio del 19\% de retorno anormal positivo para las empresas objetivo, 10 días previos al anuncio de "tender offer" para el NASDAQ.

Zingales (2000) estudió la magnitud de la prima media pagada por las acciones con derecho de voto en diferentes países (gráfico 1). Esta prima varía 
mucho entre los países, en la mayoría de ellos se sitúa entre el $10 \%$ y el $20 \%$, aunque Israel e Italia constituyen las principales excepciones. La variación se explicaría por las características propias de cada país, con una probable incidencia sobre la capacidad para extraer beneficios privados del control de las empresas. Zingales concluye que a medida que la legislación y la supervisión local avancen, las primas por control tenderán a disminuir.

Gráfico 1. Prima de acciones con derecho de voto en diferentes países.

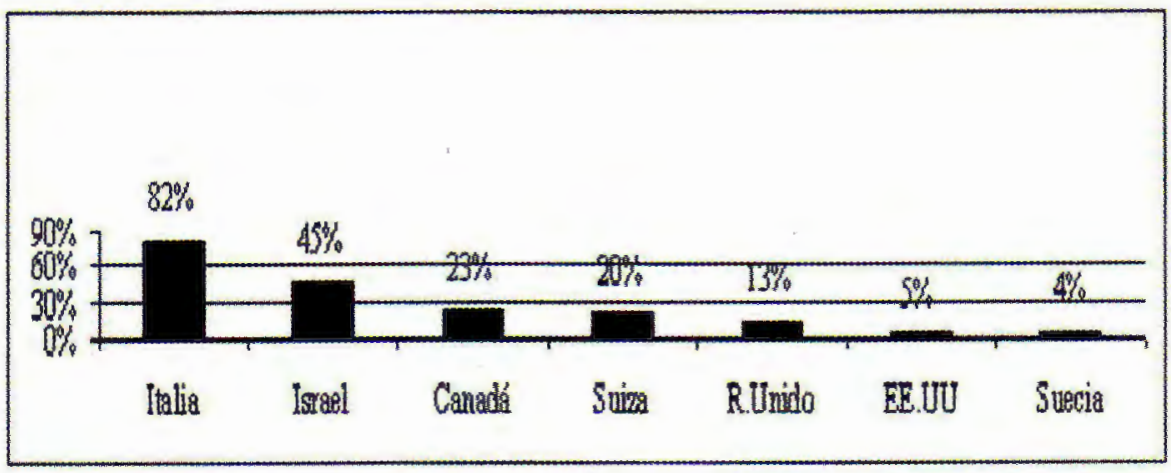

* Fuente: Zingales (2000).

Moloney (2002) investigó que en promedio después del primer anuncio de un "tender offer" (OPA), la empresa "bidder" (oferente) premia a la empresa "target" (objetivo) en un rango de 15\% a 50\% por sobre el precio de mercado de la empresa "target", antes del anuncio de la "tender offer". Concluyendo que:

- Existirá retorno anormal positivo mayor cuando son ofertas hostiles.

- Existirá un retorno anormal positivo menor cuando una propiedad tiene una concentración alta y es absorbida.

\section{Ley de OPA's}

1) El sistema que regula las Ofertas Públicas de Acciones (OPA) en Chile tiene las siguientes características:

A) Origen de la legislación: Está regulada por la Ley 19.705 del 20 diciembre de $2000{ }^{1}$

B) Antecedentes de mercados: El mercado tiene una alta concentración de la propiedad y la rotación accionaría es baja, $6.9 \%$ en el año de $2002 .{ }^{2}$ Lo que equivale a afirmar que en un año, sólo el $6.9 \%$ de la propiedad de las empresas en la bolsa cambió de dueño.

1 Ley no. 19.705 sobre Oferta Pública de Adquisición de Acciones (OPA's). Establece el régimen de gobierno corporativos. Clarke de la Cerda, A. (2001).

2 Santiago Stock Exchange-Annual Report (2002). 
C) Gatillos para informar una OPA: Cuando se pretende obtener el control de una sociedad sometida a la fiscalización de la Superintendencia de Valores y Seguros.

D) Oferta Obligatoria: Una oferta es obligatoria cuando:

I) Permite a una persona tomar el control de una sociedad.

II) Deberá someterse a OPA toda adquisición que permite alcanzar o superar el equivalente a un $30 \%$ de las acciones emitidas por una sociedad.

III) En el caso que una persona como consecuencia de una adquisición de acciones, alcance o supere los dos tercios de las acciones con derecho a voto, estará obligado a realizar una OPA por el 100\% de las acciones de la sociedad.

IV) Si una persona pretende adquirir el control de una sociedad que tiene a su vez el control de otra que haga oferta pública de adquisición de acciones de sus acciones, y que represente un $75 \%$ o más del valor de su activo consolidado, deberá efectuar previamente una oferta a los accionistas de esta última, conforme a la norma de este Título. Esta oferta deberá ser por una cantidad no inferior al porcentaje que le permita obtener el control.

V) El nuevo controlador no puede, dentro de los próximos doce meses, comprar más del $3 \%$ de las acciones si no es a través de una OPA, y el precio que se debe pagar debe ser como mínimo el de la toma de control. Pero en caso de que la compra sea en bolsa y a prorrata para el resto de los accionistas, se puede comprar un porcentaje mayor de acciones sin la necesidad de hacer una OPA.

E) Información que debe entregar:

I) Descripción financiera, jurídica y de los negocios del oferente.

II) Número de acciones o porcentaje de las acciones emitidas, cuya adquisición mínima es requisito para el éxito de la oferta.

III) Precio y condiciones de pago.

IV) Vigencia de la oferta.

V) Forma en que el oferente financiará el pago del precio de las acciones que sean adquiridas al final de la oferta.

VI) Condiciones o eventos que puedan producir la revocación de la oferta, y otras normas de carácter general de la Superintendencia.

F) Plazos en una oferta: La vigencia de la oferta no podrá ser inferior a 20 días ni superior a 30 días. Se establece la posibilidad de prorrogar la oferta por un plazo máximo de 15 días y un mínimo de 5 días.

G) Ofertas parciales. Se permite ofertas parciales, con la posibilidad de prorrateo si el número de acciones que aceptaron la oferta es mayor al número de acciones que el oferente ofreció comprar.

H) Derecho de retiro: Los accionistas que hayan entregado sus acciones pueden retractarse total o parcialmente hasta el plazo que vence la oferta o de sus prórrogas.

I) Precio: El premio por el control se relaciona con el precio sustancialmente superior al de mercado, el cual será fijado anualmente por la Súperintendencia de Valores y Seguros mediante una norma de carácter general, en un porcentaje que no puede ser inferior al $10 \%$ ni superior al $15 \%$ del precio 
de mercado de la acción. Este precio es el precio promedio ponderado de la acción tomando en consideración 90 días bursátiles antes de la fecha de la OPA y sin considerar en esto los últimos 30 días.

J) Squeeze Out: No está contemplado.

K) Artículo décimo transitorio: Es importante mencionar el artículo décimo transitorio que establece un periodo de 3 años, en que los controladores actuales podrán enajenar libremente sus acciones, aún cuando sea un precio sustancialmente superior. Se dio un periodo de 6 meses para que las empresas, a través de una junta de accionistas decidieran si se acogían a este artículo o no. Se acogieron en total 92 sociedades y no se acogieron 187.

2) Críticas al sistema que regula las Ofertas Públicas de Acciones en Chile:

A) Al establecer la obligación de realizar una OPA por todas las acciones restantes de la compañía cuando se ha alcanzado los $2 / 3$ de la propiedad, se intenta dar una puerta de escape al accionista minoritario ante la caída en la liquidez de sus títulos e intereses divergentes del controlador. Es importante recordar que con $2 / 3$ se tiene el quórum suficiente para hacer variados cambios en la empresa. La Ley de OPA's no ha hecho al mercado accionario chileno más atractivo, ni para las empresas que buscan financiamiento ni para los inversionistas. Desde el año 2000 hasta el 2002 se han cerrado 37 sociedades y sólo una se ha inscrito en la Bolsa.

B) Una Ley de OPA's equilibrada debería propender a disminuir los conflictos de intereses entre minoritario y controladores, pero también debería promover la competencia por el control corporativo con el fin de beneficiar al máximo a todos los accionistas. En la Ley de OPA's está presente el primer elemento, pero se desincentiva a los actuales controladores a vender la propiedad.

\section{Estimación de retornos anormales}

A) Metodología: Se desarrollará un estudio de evento que permitará verificar la existencia de retornos anormales positivos para las acciones de la empresa objetivo, producto del anuncio de una OPA. La medición de retornos anormales consiste en comparar los retornos obtenidos por una acción en un cierto periodo de tiempo de interés, con los retornos esperados para ese mismo periodo de tiempo, según la proyección de algún modelo estadístico. La finalidad es observar los efectos que tiene en el mercado la realización del respectivo suceso.

B) Descripción del estudio: El estudio tiene por objetivo la identificación y valorización de los retornos anormales cuando se anuncia una OPA, y se analizará en conjunto el momento y el periodo durante el cual ésta se anuncia. Eventualmente, se materializa la toma de control y otros sucesos relevantes, como conversaciones entre la empresa objetivo y la empresa oferente, utilizando la siguiente metodología:

I) Identificación de la fecha en que se anunció la OPA, $t$.

II)Definición de la ventana de evento, el cual corresponde al periodo de interés y se determina teniendo en cuenta la fecha en que se anunció una 
OPA (amuncio de la OPA ante la Superintendencia de Valores y Seguros, y a los medios de prensa). También es relevante conocer el periodo de toma de control y otros sucesos importantes, los cuales se cree que provocan rendimientos anormales en el precio de las acciones. Se considerarán tres meses de transacción bursátil, $(t-x, t+y)$, donde $x+y$ es igual a tres meses de cotización bursátil (precio de cierre) y $t$ es el momento del anuncio de una OPA, lo que permitirá medir el retorno anormal acumulado durante 3 meses de transacción bursátiles.

III)Definición de la ventana pre-evento, la cual corresponde al periodo normal previo a la ocurrencia del evento, aproximadamente a tres años de transacción bursátil.

Gráfico 2.

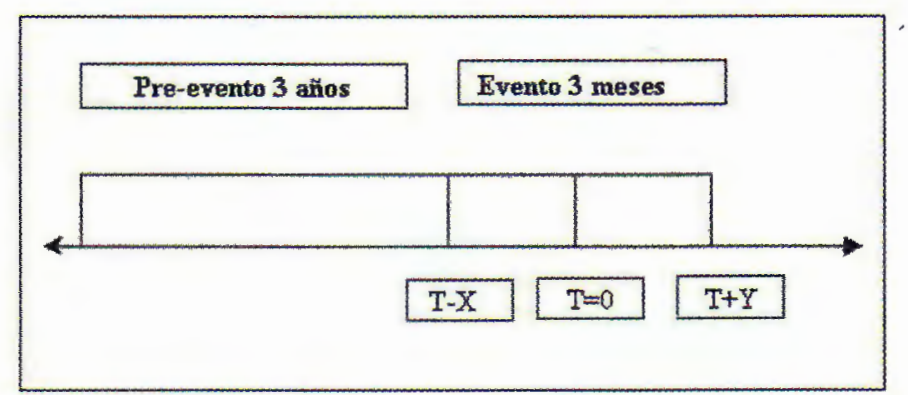

C) Criterios de selección de la muestra:

I) La muestra seleccionada comprenderá acciones que figuren en el Índice General de Precios de Acciones de la Bolsa de Comercio de Chile (IGPA) y que hayan materializado una OPA.

II) Acciones que hayan presentado un anuncio de OPA en el periodo 19952002.

III) Las acciones a evaluar deben poseer una presencia bursátil anual mayor o igual a $40 \%$ durante el periodo relevante de estudio.

IV) Presentar una estimación de $\beta$ de propiedades estadísticas adecuadas.

D) Modelos estadísticos: ${ }^{3}$ Para poder medir los retornos anormales se aplicarán tres modelos estadísticos-financieros:

I) Modelo de mercado: Incluye un coeficiente de sensibilidad ante el retorno de mercado (IGPA) debiendo cumplir el supuesto de ser distinto de 1. A través de este modelo se calculará el retorno estimado, el cual representa el rendimiento que la empresa tendría, sino se considera la OPA (periodo pre-evento tres años). Posteriormente, se calculará el error, $\epsilon_{j t}$, el cual representa la diferencia entre el retorno actual diario observado de la sociedad anónima y el retorno estimado. Lo anterior, se mide de la siguiente forma:

3 Weston, C. (2001). 


$$
R_{j t}=\alpha_{j}+\beta_{j} R M_{t}+\epsilon_{j t},
$$

donde $\epsilon_{j t}=R_{j t}-\left(\alpha_{j}+\beta_{j} R M_{t}\right)$ : Término de error que corresponde al retorno anormal para la acción $j$ en el día $t$.

$\alpha_{j}$ : Constante.

$\beta_{j}$ : Sensibilidad de la acción $j$ frente al retorno real de mercado.

$R M_{t}$ : Retorno de mercado para el día $t\left(\mathrm{IGPA}_{t}\right)$.

$\hat{R_{j t}}$ : Retorno teórico de la acción $j$ en el día $t$.

$j:$ Acción de la empresa objetivo.

$t$ : Día de medición.

$R_{j t}$ : Retorno real de la acción $j$ para el día $t$.

II) Modelo de mercado ajustado: Este modelo, a diferencia del modelo anterior, asume que el coeficiente de sensibilidad que tiene una acción específica ante el retorno de mercado, $\beta$, es igual a 1 . De tal forma que los retornos reales del IGPA en el día $t$ serán los retornos teóricos $\left(R_{j t}\right)$ de la acción $j$ en ese mismo día. Luego se calculará el residual, $\epsilon_{j t}$, el cual representa la diferencia entre el retorno actual diario observado de la firma y el retorno estimado. El modelo es el siguiente, considerando que $(\beta)=1$ :

$$
R_{j t}=R M_{t}+\epsilon_{j t},
$$

donde $\epsilon_{j t}=R_{j t}-R M_{t}$ : Término de error que corresponde al retorno anormal para la acción $j$ en el día $t$.

$R_{j t}$ : Retorno real de la acción $j$ para el día $t$.

$R M_{t}$ : Retorno de mercado para el día $t\left(\mathrm{IGPA}_{t}\right)$, correspondiente al retorno teórico de la acción.

$j$ : Acción de la empresa objetivo.

$t$ : Día de medición.

III) Modelo de media constante: Consiste en calcular la media de los retornos correspondiente al periodo de pre-evento.

$$
R_{j t}+X=\epsilon_{j t},
$$

donde $\epsilon_{j t}=R_{j t}-X$ : Término de error del retorno anormal para la acción $j$ en el día $t$.

$X$ : Media de los errores correspondiente al periodo de pre-evento.

$R_{j t}$ : Retorno real de la acción $j$ para el día $t$.

$j$ : Acción de la empresa objetivo.

$t$ : Día de medición.

E) Análisis del error: Suponiendo que tanto el modelo de predicción de retornos como la muestra son los correctos, entonces tanto los errores como su suma (es decir, los errores acumulados) deben tener una media igual a cero en la muestra de pre-evento, es decir, en una muestra considerada normal. El razonamiento detrás de esto, radica en suponer que el modelo de predicción captura todo, o gran parte del comportamiento sistemático, 
que incide en el precio de la acción. En este sentido, el término de error reflejaría los factores no sistemáticos, aleatorios, que inciden en el precio de la acción en un periodo muy breve de tiempo.

Con lo anterior, se puede concluir que: 1) Si los errores se comportan como una variable aleatoria durante el periodo del evento, entonces no podemos afirmar que existan retornos anormales durante el periodo y 2) Si lós errores no se comportan como una variable aleatoria durante el periodo del evento, entonces podemos afirmar que la acción experimenta retornos anormales, ya que los errores estarían capturando información que el modelo estadístico no captura.

F) Prueba de Error: ${ }^{4}$ Una vez obtenido el retorno teórico, de acuerdo a la ventana de estimación para las catorce sociedades de la muestra, corresponde comprobar la hipótesis principal:

Al momento de anunciarse una OPA, sobre una sociedad anónima abierta, existiría una alta probabilidad de que el mercado premie los títulos accionarios produciéndose retornos anormales positivos.

Por otro lado, también se investigarán las siguientes hipótesis secundarias:

I) Efecto de una OPA sobre una sociedad anónima abierta: Cuando el mercado ve un posible cambio estructural importante en la dirección de la empresa, debería premiar sus títulos accionarios, generando una alta probabilidad de que se produzcan retornos anormales positivos, tanto el día que se anuncia una OPA como en la acumulación de los retornos del evento.

II) Efecto de una OPA sobre una sociedad anónima abierta: Cuando el mercado no ve un cambio estructural importante en la dirección de la empresa, el precio de los títulos accionarios no debería experimentar cambios significativos, generando una alta probabilidad de que sólo se produzcan pequeños retornos anormales positivos, sólo el día que se anuncia una OPA.

III) Existe una baja probabilidad de que en las empresas que son objeto de una OPA, los retornos anormales positivos se produzcan en el periodo del evento (días antes del anuncio).

IV) Para las OPA's que se efectúen sobre sociedades acogidas al artículo 10 transitorio: El retorno ante un anuncio de una OPA será mayor que las sociedades referidas en el artículo 10 transitorio.

V) Para las OPA's que se efectúen sobre sociedades no acogidas al artículo 10 transitorio: El retorno ante un anuncio de una OPA será menor que las sociedades referidas en el artículo 10 transitorio.

Para contrastar las hipótesis se considera apropiado utilizar un nivel de confianza de $95 \%$ :

$$
\begin{aligned}
& H_{0}: \text { retornos anormales }=0, \\
& H_{1}: \text { retornos anormales } \neq 0 .
\end{aligned}
$$

4 Levin, R. (2000). 
Si se considera que los errores se distribuyen normalmente, se puede emplear la prueba $t$, ya que el estadígrafo $t$-student tiene aproximadamente una distribución normal, en el cual sólo se necesita conocer la desviación típica de los errores de la muestra pre-evento y luego comparar. Las pruebas permiten verificar si existen retornos anormales el día del anuncio. Por otro lado, debemos distinguir entre la prueba de un solo término de error y la prueba de la acumulación de tres meses de transacción bursátil.

$$
\begin{gathered}
\text { Prueba de Error Individual : } \frac{\epsilon}{\sigma_{\text {pre-evento }}}, \\
\text { Prueba de Error Acumulado }: \frac{\sum \epsilon}{\sqrt{n}\left(\sigma_{\text {pre-evento }}\right)},
\end{gathered}
$$

donde $n$ corresponde al número de errores a sumar y

$$
\frac{\epsilon}{\sigma_{\text {pre-evento }}}=\text { Error/desviación típica pre - evento. }
$$

\subsection{Verificación de resultados}

I) Si la prueba del error es positiva, se compara con el valor crítico (1.96) que corresponde a un $95 \%$ de confiabilidad. Si es mayor, se rechaza la hipótesis nula, esto implicaría que existe un retorno anormal positivo. Por otro lado, si es menor, se acepta la hipótesis nula, lo que implicaría que no existe un retorno anormal positivo.

II) $\mathrm{Si}$ el resultado de la prueba del error es negativa, se compara con el valor crítico (-1.96) que corresponde a un $95 \%$ de confiabilidad. Si es menor, se rechaza la hipótesis nula e implicaría que existe un retorno anormal negativo. Si es mayor, se acepta la hipótesis nula, lo cuall implicaría que no existe un retorno anormal negativo.

\section{Análisis de resultados}

Se analizaron catorce empresas: nueve sin legislación de OPA's, tres con legislación parcial (ya que se encontraban acogidas al artículo 10 transitorio) y dos con legislación total (ya que no se encontraban acogidas al artículo 10 transitorio).

Las conclusiones de anormalidad de los retornos son independientes de la metodología ocupada. Así, el uso de los tres modelos utilizados para estimar los retornos anormales otorgan, básicamente, los mismos resultados, lo que fortalece las conclusiones. El modelo de mercado arroja en promedio un retorno anormal al momento del anuncio de $26.5 \%$, el modelo de mercado ajustado arroja un $26.6 \%$ y el modelo de media constante un 26.9\%. Lo anterior, suponiendo normalidad y un grado de confianza de $95 \%$.

De las catorce empresas, trece presentaron un retorno anormal positivo al momento del anuncio de la OPA, lo que representa un $93 \%$ de la muestra. Respaldando la hipótesis principal de este trabajo: Al momento de anunciarse una OPA sobre una sociedad anónima abierta, existiría una alta probabilidad de 
que el mercado premie sus títulos accionarios, produciéndose retornos anormales positivos.

De las catorce empresas, seis experimentaron un retorno anormal positivo tanto al momento del anuncio como en la acumulación de los retornos. También experimentaron un retorno anormal positivo promedio al momento del anuncio mayor que el resto. El modelo de mercado arroja en promedio un retorno anormal, al momento del anuncio, de $48.9 \%$. El modelo de mercado ajustado arroja un $48.9 \%$. Y, finalmente, el modelo de media constante un $48.8 \%$. Las seis empresas presentaron grandes expectativas futuras.

Adicionalmente, se encontró evidencia de que existe mayor retorno cuando las OPA's presentan algunas de las siguientes características: A) Existe alto porcentaje de adquisición en términos de proporción de la propiedad, acompañado de una concentración baja en la propiedad absorbida, B) Existe alta competencia por el control, C) No existe legislación especial para las OPA's y D) La Ley de OPA está actuando en forma parcial. Bajo el amparo del artículo 10 transitorio no existe un límite para el premio ofrecido por la empresa "bidder" por el control corporativo.

De las catorce empresas, siete experimentaron retornos anormales positivos al momento del anuncio y una en la acumulación de los retornos. El modelo de mercado arroja en promedio un retorno anormal de $11.2 \%$, el modelo de mercado ajustado arroja un $11.4 \%$ y el modelo de media constante un $11.9 \%$, presentando las siete empresas bajas expectativas futuras. Las razones que provocaron estas pequeñas expectativas se explican porque estas OPA's cumplieron alguna o todas de las siguientes características: A) Bajo porcentaje de adquisición en términos de proporción de la propiedad, B) No existe competencia por el control, C) La propiedad absorbida tiene una concentración alta y D) Existe una legislación especial para las OPA"s.

Sólo un caso de las catorce empresas no experimentó un retorno anormal positivo al momento del anuncio, pero si en la acumulación del evento (tres meses de cotización bursátil), ya que en el mercado se especulaba con anterioridad el anuncio de la posible adquisición de la empresa norteamericana Ivax sobre el Laboratorio Chile. Existe alguna evidencia similar en los Estados Unidos, la cual presenta Asquith (1988), quien encontró un premio del $19 \%$ de retorno anormal positivo para las empresas objetivo, diez días previos al anuncio del "tender offer" para el NASDAQ.

A contimuación, se muestra un resumen de las catorce empresas investigadas. En el cuadro 1, las empresas con un asterisco experimentaron retornos anormales positivos, tanto al momento del anuncio como en la acumulación del evento. Las empresas sin asterisco sólo experimentaron retornos anormales positivos al momento del anuncio y la empresa con dos asteriscos sólo experimentó retorno anormal positivo en la acumulación de los días del evento. Después de la presentación de este cuadro, se ejemplificará tres empresas que fueron objeto de una OPA, empleando el modelo de mercado. 
Cuadro 1. Resumen de las catorce empresas investigadas.

\begin{tabular}{|c|c|c|c|c|c|c|c|c|c|}
\hline & & Empresa & Empresa & & \multicolumn{3}{|c|}{ Error al momento del anuncio } & \multirow[b]{2}{*}{ R.A. } & \multirow[b]{2}{*}{ Ley } \\
\hline Año & Mes & Objetivo & Oferente & Adq. & Mercado & Ajustado & Constante & & \\
\hline 1995 & dic. & Banco Santiago* & Gpo. Luksic* & $25.5 \%$ & $54.92 \%$ & $55.00 \%$ & $55.25 \%$ & Sí & No \\
\hline 1998 & ene. & Santa Isabel & Velox & $14.0 \%$ & $12.10 \%$ & $12.07 \%$ & $11.10 \%$ & Sí & No \\
\hline 1998 & mar. & Hábitat & Inpresa & $12.00 \%$ & $8.63 \%$ & $8.11 \%$ & $9.11 \%$ & Sí & No \\
\hline 1998 & ago. & Banco Bhif & BBV & $8.00 \%$ & $7.06 \%$ & $7.48 \%$ & $6.85 \%$ & Sí & No \\
\hline 1999 & ene. & Enersis & Endesa & $21.70 \%$ & $10.82 \%$ & $12.62 \%$ & $14.88 \%$ & Sí & No \\
\hline 1999 & ene. & Campos Chilenos $^{*}$ & Ebro Agrícola ${ }^{*}$ & $51.0 \%$ & $35.9 \%$ & $36.2 \%$ & $37.0 \%$ & Sí & No \\
\hline 1999 & ene. & Elecda & PP\& L & $19.94 \%$ & $13.92 \%$ & $13.87 \%$ & $14.88 \%$ & Sí & No \\
\hline 2000 & ago. & Banco SudAmericano* & Novoa Scotia* & $39.10 \%$ & $45.49 \%$ & $45.38 \%$ & $45.49 \%$ & Sí & No \\
\hline 2000 & nov. & Gener* & AES* $^{*}$ & $80.0 \%$ & $24.76 \%$ & $23.67 \%$ & $23.11 \%$ & Sí & No \\
\hline 2001 & may. & Laboratorio Chile** & Ivax Corporation** & $100 \%$ & $-1.16 \%$ & $-0.80 \%$ & $0.45 \%$ & No & Sí \\
\hline 2001 & jul. & Chilectra & Enersis & $2 \%$ & $11.92 \%$ & $11.75 \%$ & $11.40 \%$ & Sí & Parcial \\
\hline 2001 & ago. & $P \& S^{*}$ & Inv. Jemery Ltda. ${ }^{*}$ & $32.71 \%$ & $38.40 \%$ & $38.76 \%$ & $38.11 \%$ & Sí & Parcial \\
\hline 2002 & may. & Pampa Calichetra* & Oro Blanco* & $18.06 \%$ & $93.76 \%$ & $94.11 \%$ & $93.57 \%$ & Sí & Parcial \\
\hline 2002 & oct. & Punita de Cobre & Pacifico V Reg. S.A & $29.90 \%$ & $14.19 \%$ & $14.04 \%$ & $14.81 \%$ & Sí & Sí \\
\hline \multicolumn{4}{|c|}{ Promedio } & $32.4 \%$ & $26.5 \%$ & $26.6 \%$ & $26.9 \%$ & & \\
\hline
\end{tabular}

Fuente: Elaboración propia con datos de la Bolsa de Comercio de Santiago. Adq. significa Adquisición y R.A. significa Retorno Anormal. 
A) Análisis Banco Santiago: Por medio de este ejemplo, mostraremos el caso en donde existe retornos anormales positivos al momento del anuncio y en la acumulación de los retornos del evento. Esto ocurrió en seis casos de la muestra y representó un $42.9 \%$ del total (cuadro 1).

A.1) OPA Banco Santiago: En diciembre de 1995, el grupo Luksic anunció su intención de adquirir un 25.5\% de las acciones del Banco Santiago, a través de su sociedad de inversiones Servimotors con lo que poseería el $51 \%$ del capital social del banco.

A.2) Análisis de los errores diarios: Los errores se estimaron utilizando los tres modelos. Se mostrará el gráfico de los errores diarios obtenidos con el modelo de mercado.

Gráfico 3. Errores diarios de la acción del Banco Santiago según el modelo de mercado.

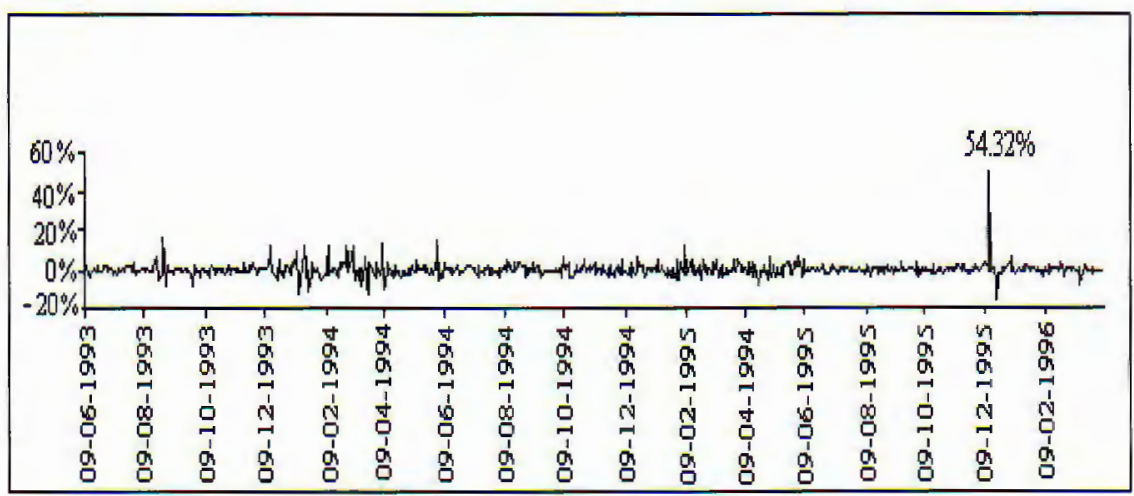

Fuente: Confeccionado con datos de la Bolsa de Comercio de Santiago.

A.3) Banco Santiago: Las conclusiones de anormalidad de los retornos son independientes de la metodología ocupada, así el uso de los tres modelos utilizados para estimar los retornos anormales otorgan básicamente los mismos resultados, lo que fortalece las conclusiones del Banco Santiago. El modelo de mercado arroja un retorno anormal al momento del anuncio de $54.92 \%$ y un retorno anormal acumulado, en los tres meses de cotización bursátil del evento, de $46.33 \%$. Por otro lado, el modelo de mercado ajustado arroja un retorno anormal al momento del anuncio de $55 \%$ y un retorno anormal en los tres meses de cotización bursátil acumulado del evento de $49.04 \%$. Finalmente, el modelo de media constante arroja un retorno anormal al momento del anuncio de $55.25 \%$ y un retorno anormal acumulado, en los tres meses de cotización bursátil del evento, de $44.61 \%$

Se puede concluir que bajo cualquier modelo y con un intervalo de confianza de $95 \%$ se rechaza la hipótesis nula. Por tal motivo, se puede afirmar que el retorno de la acción del Banco Santiago el día del anuncio, como en el acumulado obtenido de los tres meses de transacción bursátil del evento, es anormal. Lo anterior, cubre con la hipótesis principal y la primera hipótesis secundaria del presente trabajo. 
B) Análisis Santa Isabel: En este caso se presentó un retorno anormal positivo sólo al momento del anuncio. Dentro de la muestra ocurrieron siete casos, los cuales representaron un 50\% del total (cuadro 1).

B.1) OPA Santa Isabel: El 15 de enero de 1998, Disco/Ahold International, el Joint Venture conformado por Velox y Royal Ahold, anunció su intención de adquirir, mediante una oferta pública de compra de acciones y "ADS" (American Depositary Shares), el número de acciones necesarias de Santa Isabel S.A (92.533.510 títulos, representativos del $28.04 \%$ del capital) para aumentar su participación en esta sociedad a un $65 \%$.

B.2) Análisis de los errores diarios: Los errores se estimaron utilizando los tres modelos. Se mostrará en el gráfico los errores diarios obtenidos con el modelo de mercado.

Gráfico 4. Errores diarios de la acción de Santa Isabel según el modelo de mercado.

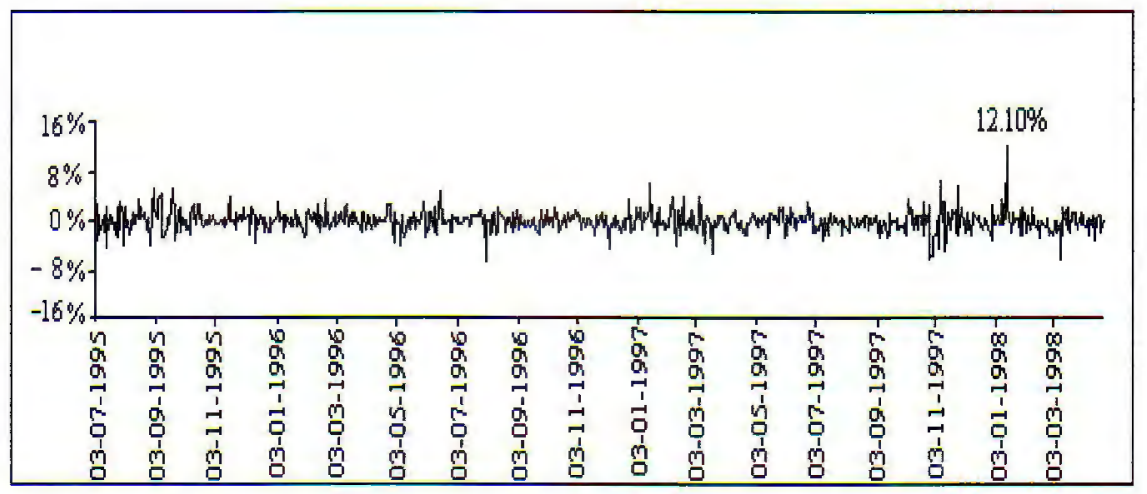

Fuente: Confeccionado con datos de la Bolsa de Comercio de Santiago.

B.3) Santa Isabel: Las conclusiones de anormalidad de los retornos son independientes de la metodología ocupada, así el uso de los tres modelos utilizados para estimar los retornos anormales otorgan básicamente los mismos resultados, lo que fortalece las conclusiones de Santa Isabel. El modelo de mercado arroja un retorno anormal al momento del anuncio de $12.10 \%$ y un retorno anormal acumulado, en los tres meses de cotización bursátil, de $14.91 \%$. Por otro lado, el modelo de mercado ajustado arroja un retorno anormal de $12.07 \%$ y un retorno anormal, en los tres meses de cotización bursátil, acumulado del evento de $17 \%$. Finalmente, el modelo de media constante arroja un retorno anormal de $11.10 \%$ y un retorno anormal acumulado, en los tres meses de cotización bursátil, de 9.,4\%.

Se puede concluir que bajo cualquier modelo y con un intervalo de confianza de $95 \%$ se rechaza la hipótesis nula para el día del anuncio. Entonces se puede afirmar que el retorno de la acción de Santa Isabel sólo el día del anuncio es anormal. Cumpliendo con la hipótesis principal de este artículo. 
C) Análisis Laboratorio Chile: Este presentó un retorno anormal positivo correspondiente a tres meses de cotización bursátil representando un $7.1 \%$ del total (cuadro 1).

C.1) OPA Laboratorio Chile: El 30 de mayo de 2001, Ivax Corporación anunció su intención de adquirir, mediante una oferta pública de compra de acciones, la totalidad de las acciones del Laboratorio Chile.

C.2) Análisis de los errores diarios: Los errores se estimaron utilizando los tres modelos. En el siguiente gráfico, se muestran los errores diarios obtenidos con el modelo de mercado, en el cual se puede observar que no se presenta un retorno anormai al momento del anuncio. Sin embargo, se presentan dos retornos anormales antes del anuncio. El primero ocurre veinte días antes de anuncio y representa un 10.50\%. El segundo aparece catorce días antes del anuncio y es igual a 7.66\%. Lo anterior, implicó que si bien no existió un retorno anormal positivo al momento del anuncio, sí existió en la acumulación de los tres meses de cotización bursátil de los días correspondientes al evento.

Gráfico 5. Errores diarios de la acción de Laboratorio Chile según el modelo de mercado.

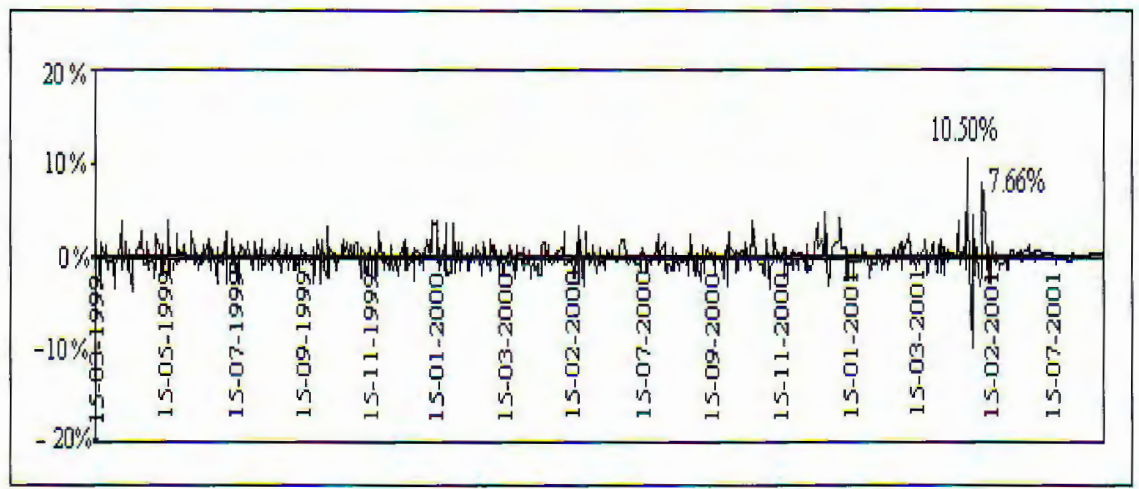

Fuente: Confeccionado con datos de la Bolsa de Comercio de Santiago.

C.3) Laboratorio Chile: Las conclusiones de anormalidad de los retornos son independientes de la metodología ocupada, así el uso de los tres modelos utilizados para estimar los retornos anormales otorgan básicamente los mismos resultados, lo que fortalece las conclusiones del Laboratorio Chile. El modelo de mercado arroja un retorno anormal al momento del anuncio de $-1.16 \%$ y un retorno anormal acumulado, en los tres meses de cotización bursátil del evento, de $20.82 \%$. El modelo de mercado ajustado arroja un retorno anormal al momento del anuncio de $-0.80 \%$ y un retorno anormal, en los tres meses de cotización bursátil acumulado del evento, de $24.70 \%$. Finalmente, el modelo de media constante arroja un retorno anormal al momento del anuncio de $0.45 \%$ y un retorno anormal acumulado, en los tres meses de cotización bursátil en los días del evento, de $28.68 \%$. 
Se puede concluir que bajo cualquier modelo y con un intervalo de confianza de $95 \%$ se rechaza la hipótesis nula para la acumulación de los días del evento. Entonces; se puede afirmar que el retorno de la acción del Laboratorio Chile fue sólo en la acumulación de los tres meses de cotización bursátil, cumpliéndose una de las hipótesis secundarias.

\section{Conclusiones}

Se han efectuado numerosos estudios en el campo de las Ofertas Públicas de las Acciones (OPA's) y premios por el control corporativo en el proceso de adquisición de la empresa objetivo. La evidencia internacional, principalmente estadounidense, ha encontrado retornos anormales positivos para la empresa objetivo de una OPA al momento de su anuncio. Se pueden destacar los trabajos de Dodd y Ruback (1977); Jarrell y Poulsen (1987); Bradley, Desai y Kim (1983); Asquith (1988); Zingales (2000) y Moloney(2002).

En esta investigación se encontraron retornos anormales positivos y significativos para las catorce sociedades seleccionadas entre el año 1995 y 2002 , las cuales fueron objeto de una OPA. Confirmando que al momento de anunciarse una OPA sobre una sociedad anónima abierta, existiría una alta probabilidad de que el mercado premie los títulos accionarios de esta última, produciendo así retornos anormales positivos. De las catorce empresas investigadas se dieron tres casos:

A) Retorno anormal positivo al momento del anuncio y en la acumulación de los retornos del evento. Esto ocurrió en seis casos representando un $42.9 \%$ del total.

B) Retorno anormal positivo sólo al momento del anuncio. Se tuvo siete casos que representaron un $50 \%$ del total.

C) Sólo existe retorno anormal positivo en la acumulación del evento correspondiente a tres meses de cotización bursátil. Se registró sólo un caso, el cual representó un $7.1 \%$ del total.

Adicionalmente, se mostró evidencia de que existe mayor retorno cuando las OPA's se distinguen por alguna o todas de las siguientes características:

A) Existe alto porcentaje de adquisición en términos de proporción de la propiedad, acompañado de una concentración baja en la propiedad absorbida.

B) Existe alta competencia por el control.

C) No existe legislación especial para las OPA's.

D) La Ley de OPA actúa en forma parcial de acuerdo con el artículo diez transitorio. Bajo el amparo de este artículo, no hay límite para el premio ofrecido por la empresa "bidder" ni por el control corporativo.

Por otro lado, las acciones de la empresa objeto de una OPA presentan retornos anormales de la acción, los cuales se explican por el aumento en el precio y por los siguientes elementos:

A) Factores especulativos antes del evento.

B) Sobreprecio que se produce en el evento. Explicado por el premio que se ofrece por el control de la empresa que es objeto de una OPA, el cual se reparte entre todos los accionistas, sean éstos mayoritarios y minoritarios. 
Se puede concluir que existe evidencia en Chile, de que al momento de anunciarse una OPA, se generan expectativas que dan como resultado retornos anormales positivos el día del anuncio, y que los retornos son menores cuando existe Ley de OPA. Por ello, se puede afirmar que, después del 1 de enero de 2004, las sociedades que sean objeto de una OPA tendrán un retorno que deberá en promedio permanecer en un 15\%, según el marco acordado por la propia Ley, y no existirán retornos cuando la Ley esté funcionado parcialmente o en casos especiales.

En el futuro, se recomienda analizar una muestra de OPA's posterior al 1 de enero de 2004, fecha en que la Ley estará funcionando completamente para todos los casos. Esto, con el propósito de comprobar que el retorno de las acciones de las empresas que son objeto de una OPA, debería situarse en un promedio menor a un $\mathbf{1 5 \%}$, según el marco acordado por la propia Ley. No existirá así, casos de acciones con retornos superiores a este promedio, lo cual ocurre cuando la Ley está funcionando parcialmente o cuando no existe legislación especial de OPA's.

\section{Bibliografía}

Asquith, D. (1988). Evidence on Theories of Volume, Bid-Ask Spreads, and Return Premia Among NASDAQ Targets of Tender Offers Bids. Doctoral Research Paper. UCLA.

Bradley, M., A. Desai, and H. Kim (1983). The Rationale behind interfirm Tender Offers. Journal of Financial Economics, 11, pp. 183-206.

Cambell, J.Y. (1996). The Econometric of Financial Markets. Princeton University Press.

Clarke de la Cerda, A. (2001). Modernización del Mercado de Capitales Chileno: La Nueva Ley de Ofertas Públicas de Adquisición de Acciones y Gobierno Corporativo. Revista Iberoamericana de Mercado de Valores.

Dimson, E. (1979). Risk Measurement when Shares are Subject to Infrequent Trading. Joumal of Financial Economics, 7, pp. 197-226.

Dodd, P. and R. Ruback (1977). Tender Offers and Stockholders Returns and Empirical Analysis. Joumal of Financial Economics, 5, pp. 351-373.

Fama, E. (1970). Efficient Capital Market: A Review of Theory and Empirical Work. Journal of Finance, 25, pp. 383-417.

Fisher, L. (1996). Some new Stock Market Indexes. Journal of Business, 39, pp. 191-225.

Jarrell, G. and A. Poulsen (1987). Shark repellents and stock prices: The effects of antitakeover amendments since 1980. Journal of Financial Economics, 19(1), pp. 127-168.

Jarrell, G., J. Brickley and J. Netter (1988). The Market for Corporate Control: The Empirical Evidence since 1980. Journal of Economic Perspective, 2(1), pp. 49-68.

Jensen, M. and R. Ruback (1983). The Market for Corporate Control. The Scientific Evidence. Journal of Financial Economics, 11, pp. 5-50.

Levin, R. (2000). Estadística para administrar. Sexta Edición. Prentice-Hall.

Moloney (2062). The Market for Corporate Control. Clemson University.

Schwert, W. (1996). Mark-up Pricing in Mergers and Acquitions. Journal of Financial Economics, 41, pp.153-192.

Weston, C. (2001). Takeover, Restructuring and Corporate Governance Siu. Prentice Hall.

Zingales, L. (2000). ¿Por qué vale la pena tener el control de la empresa? Mástering Management Diario Estrategia, 6.

Ley No. 19.705 sobre Ofẹta Pública de Adquisición de Acciones (OPA's) y Régimen de Gobierno Corporativos

Santiago Stock Exchange Arnual Report (2002). 